\title{
PAPER
}

\section{Entropy-based view of the strong field ionization process}

To cite this article: I A Ivanov et al 2019 J. Phys. B: At. Mol. Opt. Phys. 52085601

View the article online for updates and enhancements.

\section{IOP ebooks}

Bringing you innovative digital publishing with leading voices

to create your essential collection of books in STEM research.

Start exploring the collection - download the first chapter of every title for free. 


\title{
Entropy-based view of the strong field ionization process
}

\author{
I A Ivanov ${ }^{1,2}(1)$, Chang Hee Nam $^{1,3}$ and Kyung Taec Kim ${ }^{1,3}$ \\ ${ }^{1}$ Center for Relativistic Laser Science, Institute for Basic Science, Gwangju 61005, Republic of Korea \\ ${ }^{2}$ Research School of Physics and Engineering, The Australian National University, Canberra ACT 0200, \\ Australia \\ ${ }^{3}$ Department of Physics and Photon Science, GIST, Gwangju 61005, Republic of Korea \\ E-mail: igorivanov@ibs.re.kr
}

Received 17 July 2018, revised 10 December 2018

Accepted for publication 19 December 2018

Published 4 April 2019

\begin{abstract}
We apply information theoretic entropies of coordinate and velocity distributions in quantum mechanics for the description of the strong field ionization process. The approach is based on the properties of the entropies used in the information theory, viz., their ability to gauge the 'distance' between the probability distributions and thus to be sensitive to the distributions variations. Study of the entropies as functions of time allows thus to visualize conveniently evolution of the wave-function of the system undergoing strong field ionization, and to pin down, in particular, the times when the wave-function begins to change appreciably.
\end{abstract}

Keywords: Shannon entropy, strong fied ionization of atoms, tunelling and multiphoton ionization regimes

(Some figures may appear in colour only in the online journal)

\section{Introduction}

Tunneling ionization is a process occurring when atom is exposed to a strong laser field with atomic and field parameters satisfying the condition $\gamma=\omega \sqrt{2 I_{p}} / E_{0} \lesssim 1$ (Keldysh 1965, Krausz and Ivanov 2009) (here $\omega, E_{0}$ and $I_{p}$ are the frequency, electric field amplitude and ionization potential of the target system expressed in atomic units). The models introducing notion of the electron trajectory proved to be of great utility in explaining many features of this process. The well-known simple man model (SMM) (Corkum 1993, Kulander et al 1993, Lewenstein et al 1994 Krausz and Ivanov 2009, Shvetsov-Shilovski et al 2012, Arbo et al 2015), for instance, reproduces many qualitative features of the strong field phenomena, such as high harmonic generation, attosecond pulse generation and above threshold ionization.

Such models are capable to render a quantitative description of the tunneling ionization phenomena as well. The semi-classical TIPIS (tunnel ionization in parabolic coordinates with induced dipole and Stark shift) model (Pfeiffer et al 2012, Shvetsov-Shilovski et al 2012, Hofmann et al 2013), for example, has been shown to produce accurate quantitative results for the electron spectra (Pfeiffer et al 2012, Shvetsov-Shilovski et al 2012, Arbo et al 2015, Dimitrovski and Madsen 2015, Landsman and Keller 2015). In this approach, the quantum-mechanical Keldysh theory (Keldysh 1965) and its modifications (Keldysh 1965, Perelomov et al 1966, Faisal 1973, Reiss 1980, ) are used to set up initial velocities for the classical electron motion (Pfeiffer et al 2012, Arbo et al 2015, Dimitrovski and Madsen 2015). Initial value of the coordinate is defined either by the simple field direction model (FDM) or the more refined approach using the parabolic coordinate system ( $\mathrm{Hu}$ et al 1997, Landsman and Keller 2015).

Yet the question of what physical reality corresponds to this undoubtedly extremely fruitful semi-classical picture, or more precisely how we can better visualize development of the ionization process in time, remains, to some extent, open. The notion of the tunneling electron escaping from under the barrier at a given time, at some point in space, and with certain velocity, is difficult to reconcile with conventional quantum mechanics (QM). One might try to bypass the problem by escaping the conventional QM framework, using, for example Bohmian approach (Zimmermann et al 2016, Ivanov et al 2017), which reintroduces notion of the classical 
trajectory in QM. This solution may be not entirely satisfactory, the Bohmian interpretation being not universally accepted.

The so-called quantum orbits introduced by the imaginary time method (ITM) (Perelomov et al 1966, Popruzhenko 2014 b) seem to provide a basis for such a reconciliation in the framework of the conventional QM. Motion of the electron in the under-the-barrier region in the ITM approach can be visualized as a motion in the complex time with the complex velocity. Electron trajectory in the under-the-barrier region starts at a saddle-point at a complex moment of time and descends on the real axis. At the time when the trajectory crosses the real time-axis electron's coordinate and velocity (for the most probable trajectory) become real. One might then interpret this instant of time as the time of the electron's exit from under the tunneling barrier. This interpretation, however, is not entirely flawless. Because of the analyticity of the electron's action as a function of time the path connecting the saddle-point and a final moment of time when electron's velocity is measured, is not unique (Popruzhenko 2014b) and must be adequately chosen y to take into account the topology of the branch cuts arising when atomic potential is included in the theory (Popruzhenko 2014a, Pisanty and Ivanov 2016, Keil et al 2016).

More in line with the framework of the conventional QM is the point of view that all information about the system we may obtain or we may need is contained in the wave-function, the question is only how to extract it. For instance, one interpretation of the key notion in the theory of tunneling ionization- the so-called Keldysh tunneling time, is the time necessary for establishing of the static-field ionization rate when the electric field turns on instantly (McDonald et al 2013). It characterizes, therefore, the time the wave-function needs to adjust itself.

To describe an adjustment of the wave-function when electric field is present in quantitative terms we need a convenient characteristic which can be used to follow the changes the wave-function undergoes during the ionization process. We describe below the use of the information theoretic Shannon entropy (Shannon 1948) as such a characteristic. The meaning of the information entropy we need here is different from the meaning of the von Neumann entropy in QM, which provides a measure of the purity of the wave-function, and which can be used to characterize entanglement in the strong field ionization process (Majorosi et al 2017). The property of the information entropy we use is its ability to supply the space of the probability distributions with a metric (Leipnik 1959, Burbea and Rao 1982). In other words, we use information entropy to quantify the statement that one probability distribution is different from another, or, which is actually the goal of the present paper, to visualize the development of the strong field ionization process in time, using entropies as convenient measures illustrating evolution of the wave-function. We will study below entropies of the coordinate and velocity probability distributions for the process of strong field ionization. Entropy of a probability distribution $f$ is its integral characteristic assigning a number $S(f)$ - the entropy of the distribution, to the given distribution. Evolution of the entropies in time is, therefore, easier to follow, visualize and interpret than evolution of the wave-function. The correspondence $f \rightarrow S(f)$, as we shall see, preserves many important features of the ionization process, and allows to illustrate them in a clear and concise manner.

\section{Theory}

Using the definition of the theoretic Shannon entropy (Shannon 1948), entropies of the coordinate and velocity distributions $\left(S_{x}(t)\right.$, and $S_{v}(t)$, respectively), can be defined as:

$$
\begin{aligned}
& S_{x}(t)=-\int|\Psi(\boldsymbol{r}, t)|^{2} \log |\Psi(\boldsymbol{r}, t)|^{2} d \boldsymbol{r}, \\
& S_{v}(t)=-\int|\tilde{\Psi}(\boldsymbol{q}, t)|^{2} \log |\tilde{\Psi}(\boldsymbol{q}, t)|^{2} d \boldsymbol{q} .
\end{aligned}
$$

Here $\Psi(\boldsymbol{r}, t)$ is the coordinate wave-function describing the system, $\tilde{\Psi}(\boldsymbol{q}, t)$ its Fourier transform. Both $\Psi(\boldsymbol{r}, t)$ and $\tilde{\Psi}(\boldsymbol{q}, t)$ are not dimensionless quantities so, strictly speaking, logarithms of these quantities in equation (1) are not well defined. For these logarithms to make sense we could choose a parameter $c_{x}$ having the physical dimension of $|\Psi(\boldsymbol{r}, t)|^{2}$, and a parameter $c_{v}$ having the physical dimension of $|\tilde{\Psi}(\boldsymbol{q}, t)|^{2}$, and replace the logarithms in (1) with $\log \left(|\Psi(\boldsymbol{r}, t)|^{2} / c_{x}\right)$ and $\log \left(|\tilde{\Psi}(\boldsymbol{q}, t)|^{2} / c_{v}\right)$. The entropies would then become functions $S_{x}\left(t, c_{x}\right), S_{v}\left(t, c_{v}\right)$ of these parameters . One can easily see that since both coordinate and momentum wave-functions are normalized to unity and remain normalized in the course of the evolution, for the entropies obtained using different sets of parameters $c^{(1)}$ and $c^{(2)}$ one has $S\left(t, c^{(2)}\right)-S\left(t, c^{(1)}\right)=\log \left(c^{(2)} / c^{(1)}\right)$. The coordinate and velocity entropies are, therefore, defined by the equation(1) only up to an arbitrary additive factors (the property they share with the statistical entropy in the classical physics (Landau and Lifshitz 1980)). If, as we will do below, we are interested in the entropy change, this arbitrary factors become immaterial, and we can just choose them to be 1 both in the coordinate and the momentum spaces.

We note that the definitions of $S_{x}$ and $S_{v}$ in equation (1) apply in both length $(L)$ and velocity $(V)$ gauges we may use to describe atom-field interaction (Sobelman 1972). Obviously, $S_{x}$ does not depend on the choice of the gauge since transformation (we assume dipole approximation) $\Psi_{V}(\boldsymbol{r}, t)=e^{-i \boldsymbol{A}(t) \cdot \boldsymbol{r}} \Psi_{L}(\boldsymbol{r}, t)$ from the $L$-to the $V$-gauge does not affect $|\Psi(\boldsymbol{r}, t)|^{2}$. In the case of the velocity distribution, velocity $\boldsymbol{v}$ in the $V$-gauge is related to the wave-vector $\boldsymbol{q}$ of the Fourier transform $\tilde{\Psi}(\boldsymbol{q}, t)$ as $\boldsymbol{v}=\boldsymbol{q}+\boldsymbol{A}(t)$. Therefore, velocity distribution in the $V$-gauge is given by $|\tilde{\Psi}(\boldsymbol{v}-\boldsymbol{A}(t), t)|^{2}$, and the integral defining $S_{v}(t)$ can still be written in the form equation (1) by shifting the dummy integration variable.

Below we will study the evolution of $S_{x}(t)$ and $S_{v}(t)$ with time for the process of strong field ionization of hydrogen atom. The coordinate wave-function $\Psi(\boldsymbol{r}, t)$ describing the ionization process is obtained by solving the time-dependent Schrödinger equation (TDSE) for a hydrogen atom in 
presence of a laser pulse:

$$
i \frac{\partial \Psi(\boldsymbol{r})}{\partial t}=\left(\hat{H}_{\mathrm{atom}}+\hat{H}_{\mathrm{int}}(t)\right) \Psi(\boldsymbol{r}),
$$

with $\hat{H}_{\text {atom }}=\frac{\hat{\boldsymbol{p}}^{2}}{2}-\frac{1}{r}$, and the length gauge form $\hat{H}_{\text {int }}(t)=\boldsymbol{E}(t) \cdot \hat{\boldsymbol{r}}$ for the interaction operator. We will follow evolution of the hydrogen atom initially in the ground state with the wave-function $\phi_{0}$ and energy $\epsilon_{0}=-0.5$ a.u. To solve the TDSE numerically we follow the procedure described in detail in our earlier works (Ivanov and Kheifets 2013, Ivanov 2014). We will present below, therefore, only the most essential details of the calculation.

The electric field of the laser pulse is linearly polarized (along the $z$-axis, which we use also as the quantization axis) and is defined in terms of the vector potential $\boldsymbol{E}(t)=$ $-\frac{\partial \boldsymbol{A}(t)}{\partial t}$, where:

$$
\boldsymbol{A}(t)=-\hat{z} \frac{E_{0}}{\omega} \sin ^{2}\left\{\frac{\pi t}{T_{1}}\right\} \sin \omega t,
$$

with the electric field amplitude $E_{0}$, carrier frequency $\omega$, and total duration $T_{1}=N T$, where $T=2 \pi / \omega$ is an optical cycle (o.c.) corresponding to the frequency $\omega$ and $N$ is an integer. For a given pulse duration $T_{1}=N T$ we consider evolution of the system on the interval $(0,(N+1) T)$, allowing one optical cycle of the field-free evolution of the system after the end of the pulse. We will consider pulses with the fixed pulse duration of $N=2$, and we will vary the carrier frequency $\omega$ and the electric field amplitude $E_{0}$. The corresponding pulse shapes are shown in figure 1.

The solution of the TDSE is represented as a series in spherical harmonics:

$$
\Psi(\boldsymbol{r}, t)=\sum_{l=0}^{l_{\max }} \frac{f_{l}(r, t)}{r} Y_{l 0}(\hat{\boldsymbol{r}}) .
$$

Radial variable is discretized on the grid with the stepsize $\delta r=0.1$ a.u. in a box of the size $R_{\max }$. We used $R_{\max }=600$ a.u. in the calculations below. As for the parameter $l_{\max }$ in equation (4), its optimal value depends on the base frequency of the laser pulse, growing with decreasing frequency. Necessary accuracy checks have been performed to ensure that convergence of the expansion (4) has been achieved in the calculations. For the smallest frequency $\omega=0.03$ a.u. we consider below we had to use $l_{\max }=70$.

\section{Results}

In figures 1 and 2 we show the entropies of the coordinate and momentum distributions for different ionization regimes corresponding to different driving pulse frequencies. We see clearly distinct behavior of the entropies. With decreasing frequency the entropies begin to exhibit sharp variations at times close to the local maxima of the field, justifying the expectations that entropies can capture the instant of time when distributions begin to change. These variations are characteristic not only of the tunneling regime of ionization.
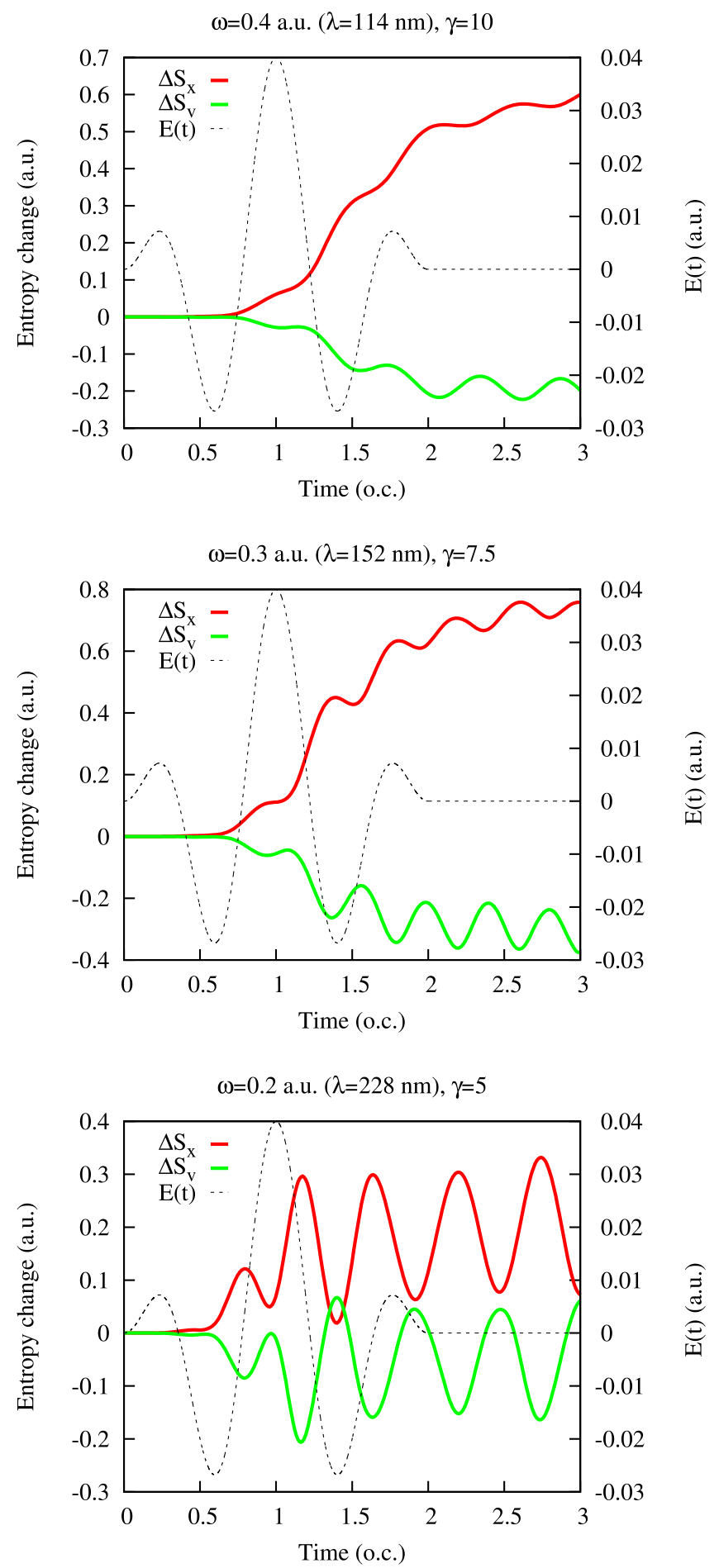

Figure 1. Entropies of the coordinate and velocity distributions for a driving pulse with the electric field amplitude $E_{0}=0.04$ a.u. in the multiphoton ionization regime.

They can appear also in the multiphoton regime as figure 3 shows. The difference between the multiphoton regime in figures 1 and 3 is the large value of the multiquantum parameter $K=I_{p} / \omega$ in the case of the data shown in figure 3 . We cannot expect a complete similarity of the data for equal $\gamma$-values if values of $K$ are different, at least two dimensionless parameters (e.g. $\gamma$ and $K$, or $\gamma$ and the reduced 

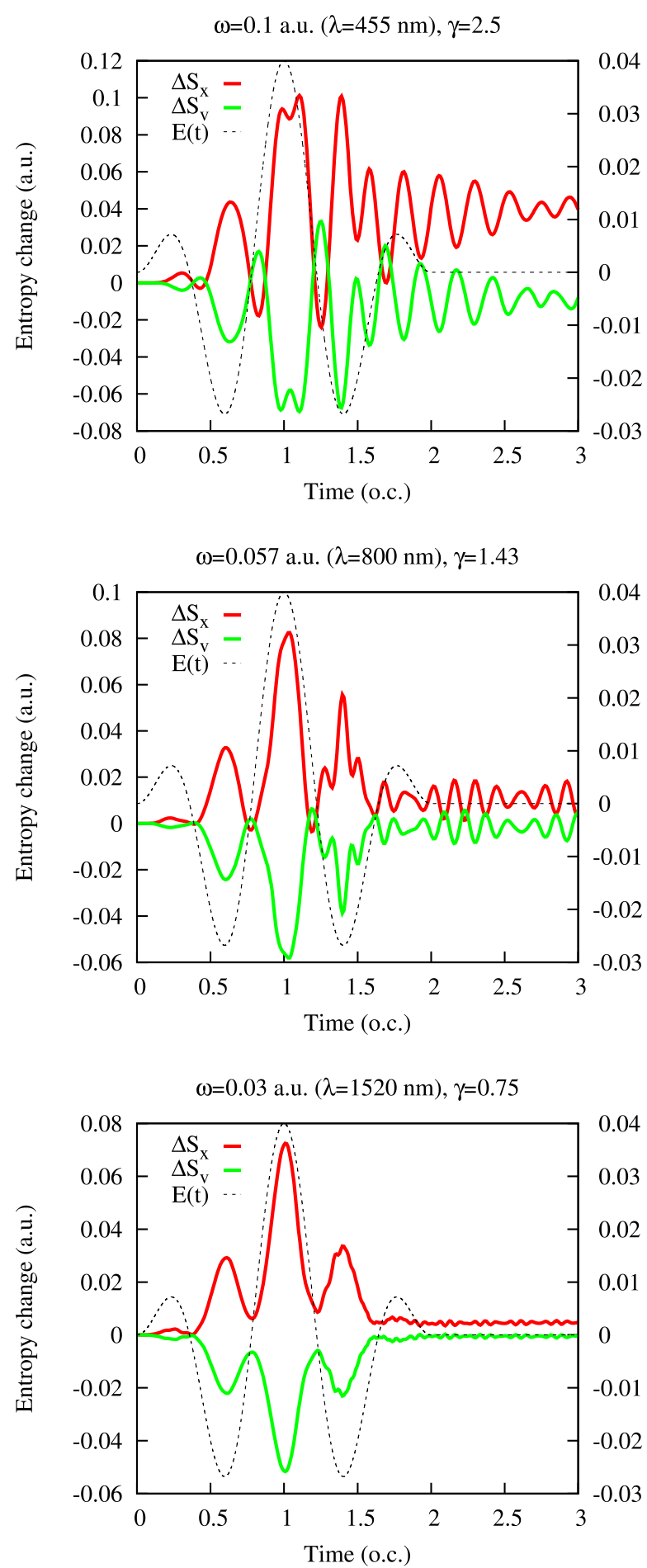

Figure 2. Entropies of the coordinate and velocity distributions for a driving pulse with the electric field amplitude $E_{0}=0.04$ a.u. in the tunneling ionization regime.

electric field $E_{0} /\left(2 I_{p}\right)^{3 / 2}$ ) are needed to describe the ionization process (Keil et al 2016). The presence of the sharp variations of the entropies near the field maxima for large values of the $K$-parameter both in figures 2 and 3 suggests that these variations are due to the high degree of nonlinearity of the ionization process for the large $K$ values.

Equation (1) defining entropies is nonlinear and rather difficult to analyze. We will describe below some approximations,

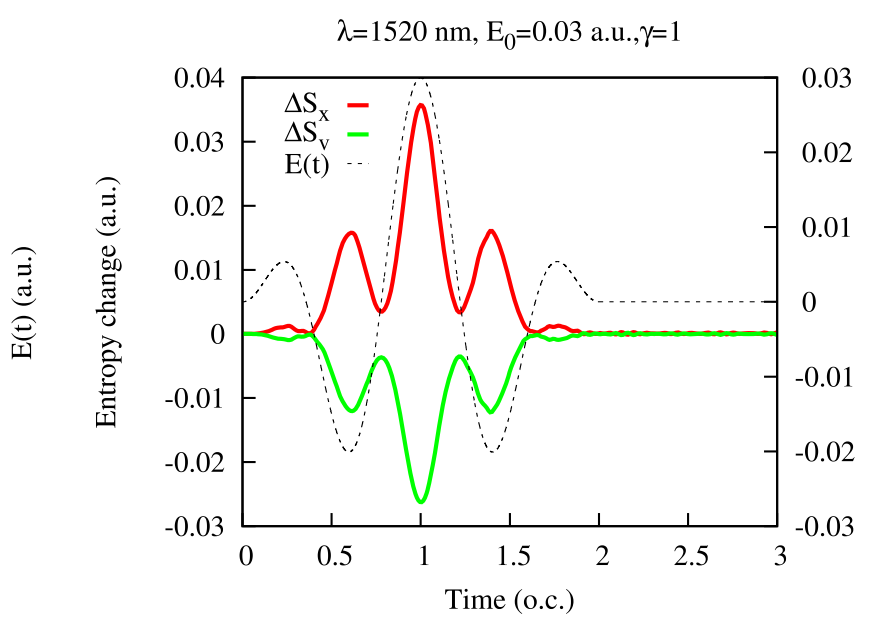



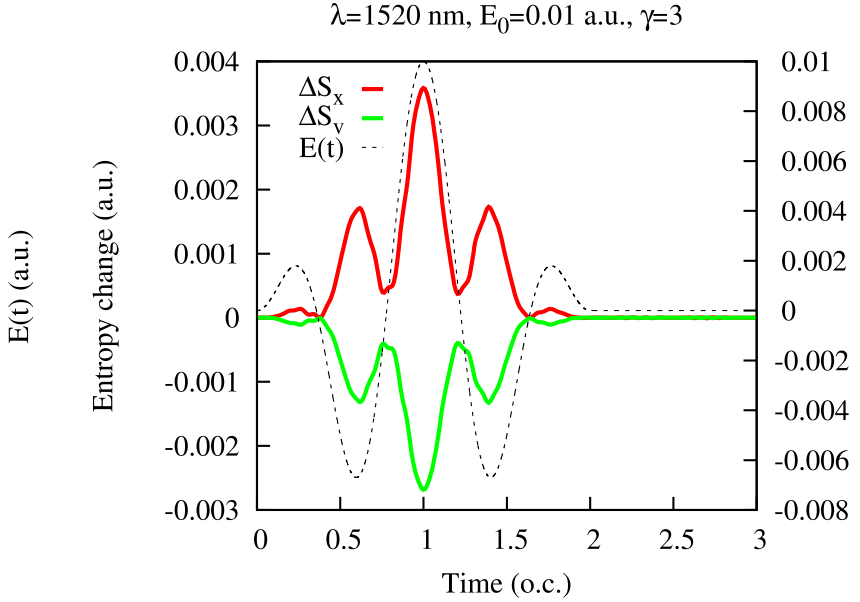

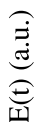



Figure 3. Entropies of the coordinate and velocity distributions for a driving pulse with base frequency $\omega=0.03$ a.u. for different electric field amplitudes.

which help to understand qualitatively the behavior of the entropies shown in figures 1,2 and 3.

Let us represent the time-dependent wave-function describing the evolution of the system as:

$$
\Psi(t)=e^{-i \epsilon_{0} t} \phi_{0}+\Psi_{1}(t),
$$

where $\phi_{0}$ is the initial ground atomic state with energy $\epsilon_{0}$. The equation (5) serves as a definition of the wave-packet $\Psi_{1}(t)$. It 
is convenient to further decompose $\Psi_{1}(t)$ in the mutually orthogonal components:

$$
\begin{aligned}
\Psi_{1}(t) & =\Psi_{\mathrm{ion}}(t)+\Psi_{\mathrm{ex}}(t)+\Psi_{\mathrm{gs}}(t) \\
& =\Psi_{2}(t)+\Psi_{\mathrm{gs}}(t),
\end{aligned}
$$

with $\Psi_{2}(t)=\Psi_{\text {ion }}(t)+\Psi_{\mathrm{ex}}(t)$. These components are obtained by projecting $\Psi_{1}(t)$ on the continuous spectrum of the field-free atomic Hamiltonian (in the case of $\Psi_{\text {ion }}(t)$ ), on the subspace spanned by all excited bound states of the hydrogen atom (for $\Psi_{\mathrm{ex}}(t)$ ), and on the subspace defined by the ground state of the hydrogen atom (for $\Psi_{\mathrm{gs}}(t)$ ).

Our goal is to use the the decomposition (5) of the total wave-function as a starting point for the perturbation expansion for the entropies. For this perturbative approach to work we have to show that $\left\|\Psi_{1}(t)\right\|^{2} \ll 1$ in the course of the evolution. To quantify the statement $\left\|\Psi_{1}(t)\right\|^{2} \ll 1$ in detail we show in figure 4 the squared norm of the wave-packet $\Psi_{1}(t)$ for the typical field parameters we employ in the calculations for different values of the multiquantum parameter $K$ and Keldysh parameter $\gamma$. For the reader's convenience we show also the norms of the mutually orthogonal components into which $\Psi_{1}$ has been decomposed in equation (6). To avoid confusion, we note that only the norms of the $\left|\Psi_{\text {ion }}(t)\right|^{2}$ and $\left|\Psi_{\mathrm{ex}}(t)\right|^{2}$ remain constant after the end of the pulse, the norm of the $\Psi_{1}(t)$, as defined in the equation (5), generally varies even for the field-free evolution. As figure 4 shows the norm of $\Psi_{1}(t)$ satisfies $\left\|\Psi_{1}(t)\right\|^{2} \lesssim 0.025$ for all the interval of the pulse duration, thus justifying the applicability of the perturbative approach. As to the physical meaning of norms of the components into which we decomposed $\Psi_{1}(t)$ above, we should emphasize, that this decomposition acquires its full physical meaning only after the end of the laser pulse. The squared norms $\left|\Psi_{\text {ion }}(t)\right|^{2}$ and $\left|\Psi_{\text {ex }}(t)\right|^{2}$ become, after the end of the pulse, the ionization and excitation probabilities, respectively. Inside the interval of the pulse duration, we cannot assign this physical meaning to $\left|\Psi_{\text {ion }}(t)\right|^{2}$ and $\left|\Psi_{\text {ex }}(t)\right|^{2}$ quite unambiguously. This can be seen, e.g. from the fact that the separation (6) of the wave-function for the times inside the interval of the laser pulse duration is not gauge invariant. It might be more correct to talk not about the excitation and ionization processes for the times inside the laser pulse, but about the norms of the wave-packets $\left|\Psi_{\text {ion }}(t)\right|^{2}$ and $\left|\Psi_{\mathrm{ex}}(t)\right|^{2}$. We will, however, use these terms interchangeably, even for the times inside the laser pulse duration, which, we hope, will not lead to a confusion. As one can see from the figure 4 and the corresponding plot in the figures 1,2 , the coordinate entropy largely mimics the behavior of the norms of the excitation and ionization probabilities. The reason for that is the highly nonlinear character of the ionization process for the large values of $K$. We should emphasize, however, that unlike the notions of the excitation and ionization for the times inside the interval of the laser pulse duration, the entropies are perfectly well-defined quantities which, therefore, can be used to follow the ionization process without any ambiguities.

At the moment we are more concerned with the mathematical aspect of the problem. The statement $\left\|\Psi_{1}(t)\right\|^{2} \ll 1$ remains true for the weak enough fields we consider regardless of the choice of the gauge we may employ to describe $\lambda=1520 \mathrm{~nm}, \mathrm{E}_{0}=0.03$ a.u., $\gamma=1, \mathrm{~K}=15.2$

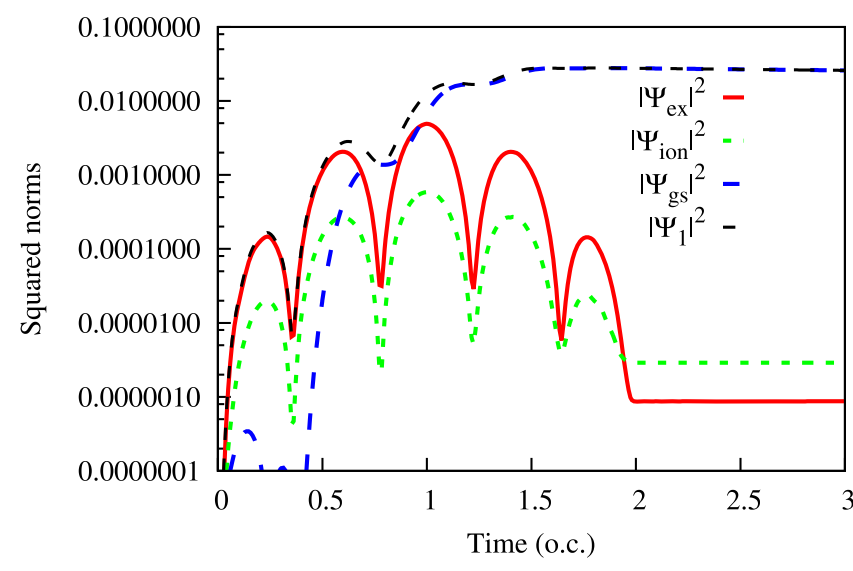

$\lambda=228 \mathrm{~nm}, \mathrm{E}_{0}=0.04$ a.u. $, \gamma=5, \mathrm{~K}=2.5$

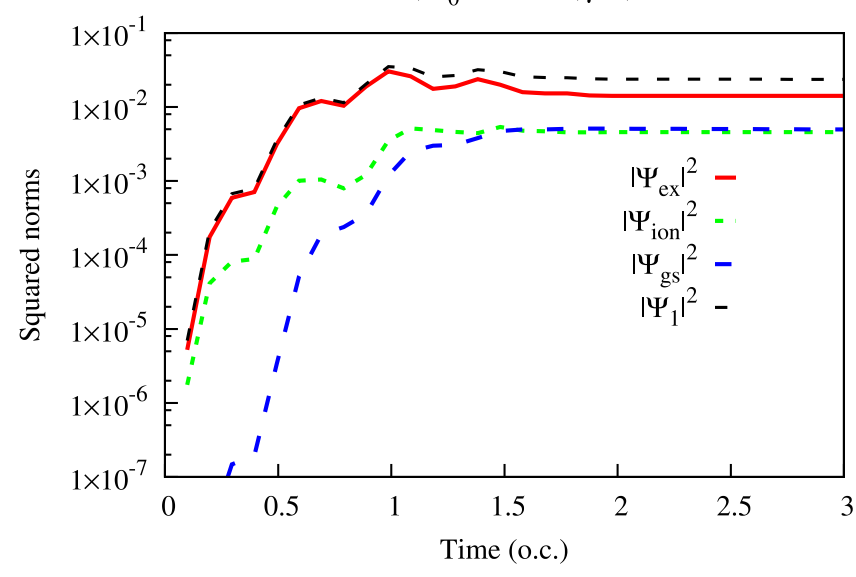

$\lambda=1520 \mathrm{~nm}, \mathrm{E}_{0}=0.005$ a.u., $\gamma=6, \mathrm{~K}=15.2$

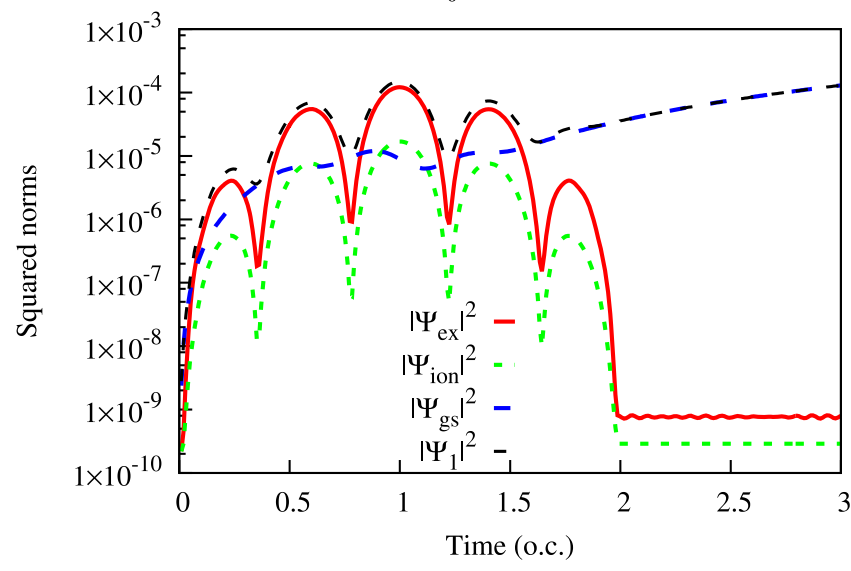

Figure 4. Norms of the various components of the wave-packet $\Psi_{1}(t)$ as functions of time for different $\gamma$ and $K$ values.

atom-field interaction. We will use this fact to develop a perturbative approach to the calculation of entropies, which will allow us to get a more clear understanding of the entropies behavior. A detailed study of all the ionization regimes, for different $\gamma$ and $K$ values being hardly possible in a single publication, we will concentrate below on the case of the tunneling ionization for the field parameters used to obtain the data in the top panel of the figure $4(\omega=0.03$ a.u. $E_{0}=0.03$ a.u.). 

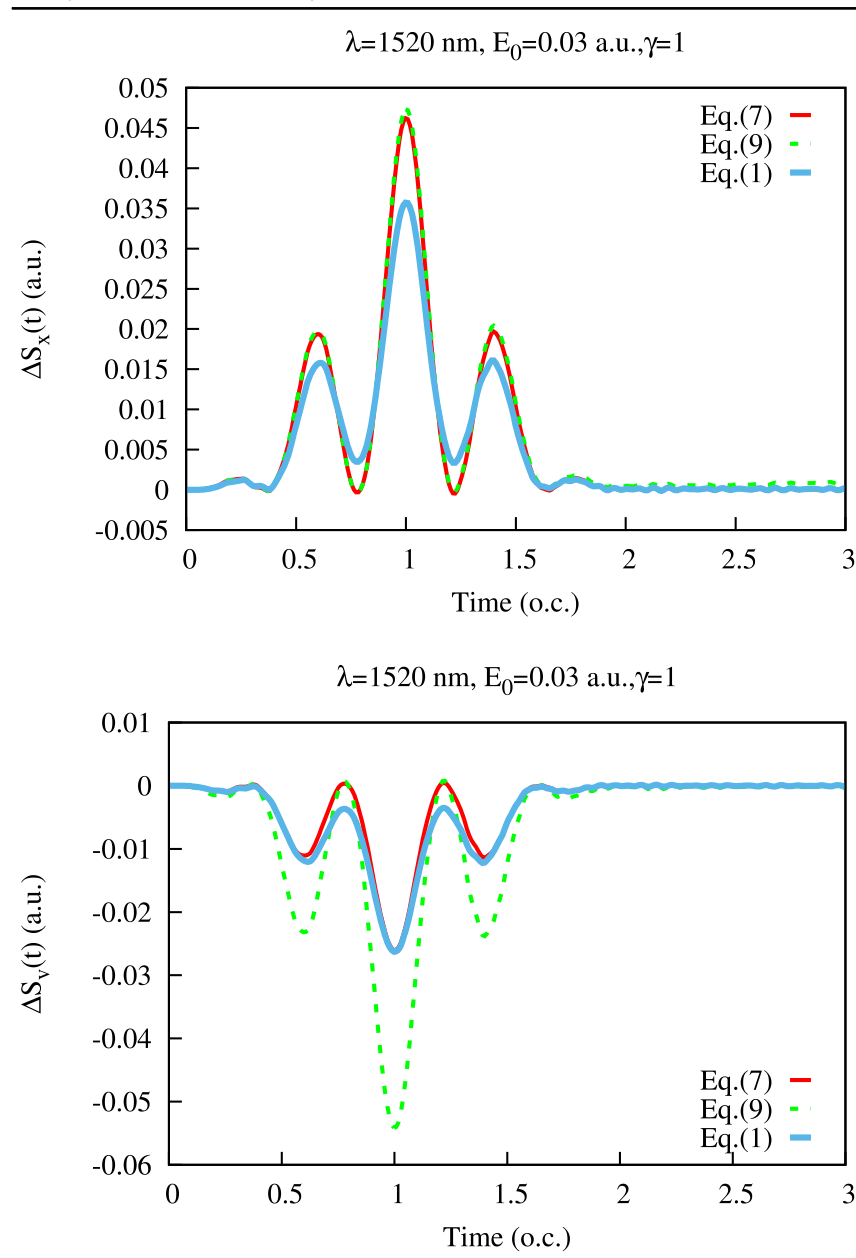

Figure 5. Results of the linearized (equation (7)) and asymptotic (equation (9)) approximations.

Let us consider the entropies changes $\Delta S_{x}(t)=$ $S_{x}(t)-\mathrm{S}_{x}(0)$ and $\Delta S_{v}(t)=S_{v}(t)-\mathrm{S}_{v}(0)$ induced by the field. Here, $S_{x}(0)$ and $S_{v}(0)$ are coordinate and velocity entropies of the field-free hydrogen atom in the ground state (these values can easily be calculated and are $S_{x}(0)=3+\log \pi \approx 4.15$, $\left.S_{v}(0) \approx 2.42\right)$. Expanding the integrand in equation (1) using $u \log u \approx u_{0} \log u_{0}+\left(u-u_{0}\right)\left(1+\log u_{0}\right)$, with $u=|\Psi(t)|^{2}$, $u_{0}=\left|\phi_{0}\right|^{2}$, and $u-u_{0}=|\Psi(t)|^{2}-\left|\phi_{0}\right|^{2} \approx 2 \operatorname{Re} e^{i \epsilon_{0} t} \phi_{0}^{*} \Psi_{1}(t)$ and keeping only the terms up to the first order in the small $\Psi_{1}(t)$, we obtain:

$$
\begin{aligned}
\Delta S_{x}(t)= & -2 \operatorname{Re} e^{i \epsilon_{0} t} \int \phi_{0}^{*}(\boldsymbol{r}) \Psi_{1}(\boldsymbol{r}, t) \\
& \times \log \left(e\left|\phi_{0}(\boldsymbol{r})\right|^{2}\right) d \boldsymbol{r}+O\left(\left\|\Psi_{1}(\boldsymbol{r}, t)\right\|^{2},\right. \\
\Delta S_{v}(t)= & -2 \operatorname{Re} e^{i \epsilon_{0} t} \int \tilde{\phi}_{0}^{*}(\boldsymbol{q}) \tilde{\Psi}_{1}(\boldsymbol{q}, t) \\
& \times \log \left(e \mid \tilde{\phi}_{0}\left(\left.\boldsymbol{q}\right|^{2}\right) d \boldsymbol{q}+O\left(\left\|\tilde{\Psi}_{1}(\boldsymbol{q}, t)\right\|^{2} .\right.\right.
\end{aligned}
$$

The tilted quantities in equation (7) stand for the Fourier transforms of the corresponding coordinate functions. The accuracy of the linearized approximation (7) can be judged from the figure 5 .

To progress further, we note that the logarithmic factors in equation (7) vary slowly in the coordinate and momentum spaces. Wave-packet $\Psi_{2}(\boldsymbol{r}, t)$ defined in equation (6) and its Fourier transform $\tilde{\Psi}_{2}(\boldsymbol{q}, t)$, on the other hand, vary fast in the
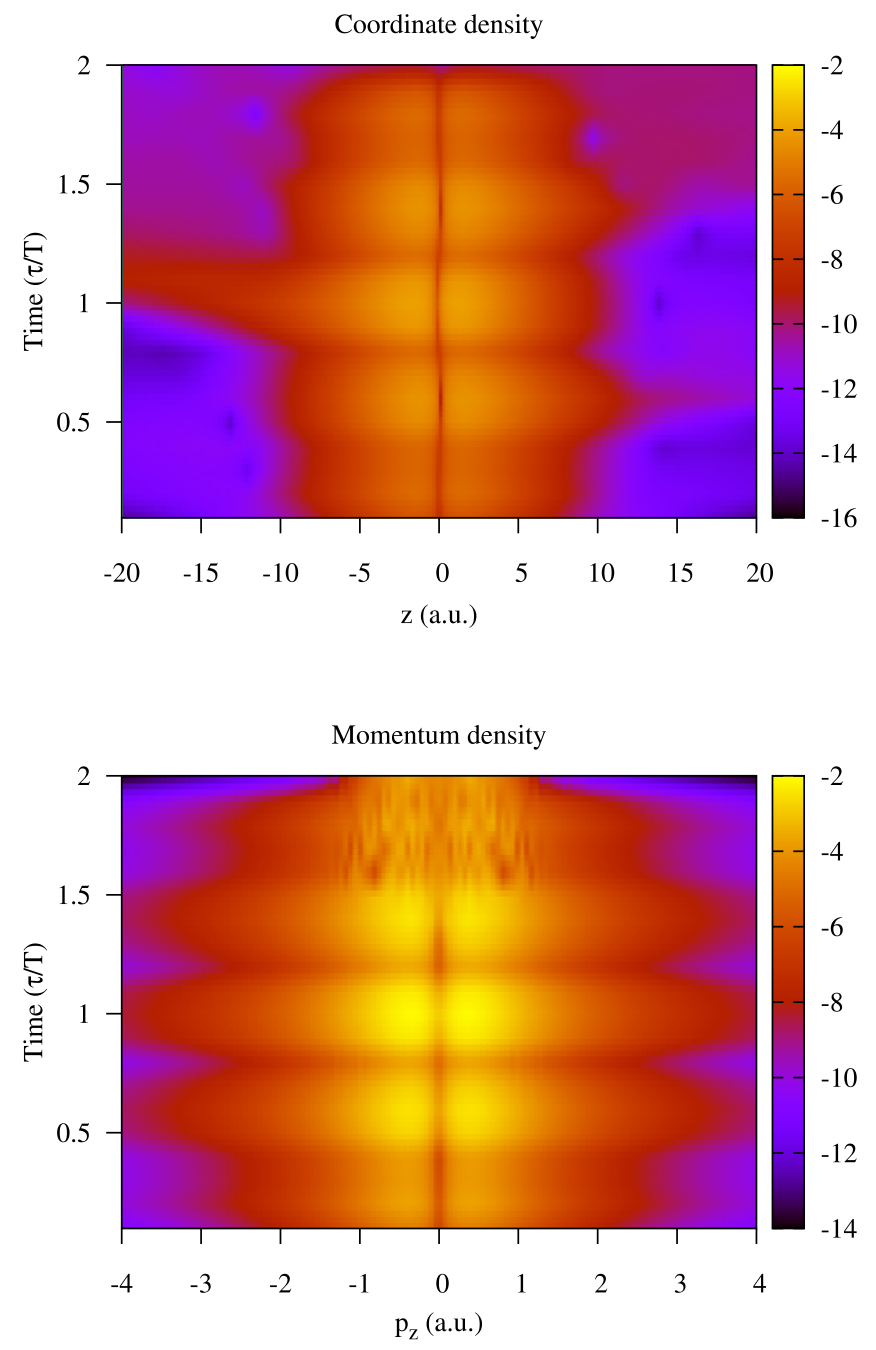

Figure 6. The coordinate and the momentum densities shown are the quantities $\log _{10}\left|\Psi_{2}(0,0, z, t)\right|^{2}$ and $\log _{10}\left|\tilde{\Psi}_{2}\left(0,0, p_{z}, t\right)\right|^{2}$ as functions of time for the pulse with $E_{0}=0.03$ a.u. $\omega=0.03$ a.u.

coordinate and momentum spaces, correspondingly. To illustrate this statement, we show in figure 6 the evolution in time of the coordinate density $\left|\Psi_{2}(0,0, z, t)\right|^{2}$, and the momentum density $\left|\tilde{\Psi}_{2}\left(0,0, p_{z}, t\right)\right|^{2}$ along the direction of the polarization vector. To show more structure, which would be obscured had we used the linear scale, we use the logarithmic scale in figure 6.

It is worthwhile to compare the behavior of the coordinate density in figure 6 and the norms of the wave-packets $\left|\Psi_{\text {ion }}(t)\right|^{2}$ and $\left|\Psi_{\text {ex }}(t)\right|^{2}$ shown in the figure 4 which we discussed above. The coordinate and the momentum densities in figure 6 vary in time and reach maximum value every half cycle following a similar pattern in figure 4 . Let us take the interval around $t=1$ o.c. (the main maximum of the electric field) as an example. Horizontal slice of the top panel of the figure 6 , taken at $t=1$ o.c., shows a structure consisting of several components. We can understand these structures qualitatively using the resolution $\Psi_{2}(t)=\Psi_{\text {ion }}(t)+\Psi_{\text {ex }}(t)$ of the wave-packet $\Psi_{2}(t)$ into the excitation and ionization parts we introduced in equation (6) We can interpret the maxima of this structure located at $|z| \approx 1-2$ a.u. as the peaks due to 
the excitation part $\Psi_{\mathrm{ex}}(t)$ of the wave-packet. The sleeve beginning at $z \approx-10$ a.u. can be attribute to the 'ionized' part $\Psi_{\text {ion }}(t)$ of the wave-packet for the laser pulse we consider electrons can tunnel out in the negative $z$-direction at times close to $t=1$ o.c. These interpretations are, of course, of a qualitative character only. As we mentioned above, the different terms in the decomposition equation (6) of the wavepacket $\Psi_{1}(t)$ into its excitation, ionization, and the ground state parts, acquire the precise physical meaning only after the end of the pulse.

The momentum density plot shown in the bottom panel of figure 6 shows maxima which can again be attributed to the excitation part of the wave-packet in the momentum space. One can also discern a ragged structure, which becomes dominant after $t \gtrsim 1.5$ o.c. and which defines the momentum distribution after the end of the pulse. This ragged structure is due to the ionized wave-packet. Such structures are known to arise in the longitudinal momentum distributions because of the quantum interference (Dimitriou et al 2004).

Our aim in showing the densities in figure 6 was to show that the wave-packets $\Psi_{2}(\boldsymbol{r}, t)$ and $\tilde{\Psi}_{2}(\boldsymbol{q}, t)$ vary fast comparing to the logarithmic factors in the equation (7). Both coordinate and momentum distributions in figure 6 indeed change considerably on a scale of an atomic unit, both in the momentum and coordinate spaces. The same observation applies to the part $\Psi_{\mathrm{gs}}(t)$ of the wave-packet in equation (6) The well -known expressions for the coordinate and momentum space wave-functions of the hydrogen atom $\phi_{0}(\boldsymbol{r})=\frac{1}{\sqrt{\pi}} e^{-r}, \tilde{\phi}_{0}(\boldsymbol{q})=\frac{\sqrt{8}}{\pi\left(1+q^{2}\right)^{2}}$ (Landau and Lifshitz 1977) certainly vary more slowly in the coordinate and momentum spaces than their logarithms.

For the estimate of the integrals in equation (7) we can use, therefore, the well-known recipe (Fedoryuk and Shabunin 1985), often employed for the asymptotic analysis of integrals. We can replace the slowly varying logarithmic factors in equation (7) with their small $-r$ and small $-p$ asymptotics respectively. These asymptotics are: $\log \left(e\left|\phi_{0}(\boldsymbol{r})\right|^{2}\right) \approx c_{1}-2 r, \log \left(e \mid \tilde{\phi}_{0}\left(\left.\boldsymbol{q}\right|^{2}\right) \approx c_{2}-4 q^{2}\right.$, where $c_{1}=1, c_{2}=1+\log 8-2 \log \pi$.

Substituting these small $-r$ and small- $p$ expansions in the equation (7) we obtain:

$$
\begin{aligned}
\Delta S_{x}(t)= & -2 \operatorname{Re} e^{i \epsilon_{0} t} \int \phi_{0}^{*}(\boldsymbol{r}) \Psi_{1}(\boldsymbol{r}, t)\left(c_{1}-2 r\right) \\
& \times d \boldsymbol{r}+O\left(\left\|\Psi_{1}(\boldsymbol{r}, t)\right\|^{2},\right. \\
\Delta S_{v}(t)= & -2 \operatorname{Re} e^{i \epsilon_{0} t} \int \tilde{\phi}_{0}^{*}(\boldsymbol{q}) \tilde{\Psi}_{1}(\boldsymbol{q}, t)\left(c_{2}-4 q^{2}\right) \\
& \times d \boldsymbol{q}+O\left(\left\|\tilde{\Psi}_{1}(\boldsymbol{q}, t)\right\|^{2} .\right.
\end{aligned}
$$

The integral $\int \phi_{0}^{*}(\boldsymbol{r}) r \Psi_{1}(\boldsymbol{r}, t) d \boldsymbol{r}$ in the (8) for the coordinate entropy change is of the order of $O\left\|\Psi_{1}\right\|$. To estimate the integral $a(t)=\int \phi_{0}^{*}(\boldsymbol{r}) \Psi_{1}(\boldsymbol{r}, t) d \boldsymbol{r}$ in the equation (8) we substitute for $\Psi_{1}(\boldsymbol{r}, t)$ the decomposition (6): $\Psi_{1}(\boldsymbol{r}, t)=$ $\Psi_{2}(\boldsymbol{r}, t)+\Psi_{\mathrm{gs}}(\boldsymbol{r}, t)$. The integral $\int \phi_{0}^{*}(\boldsymbol{r}) \Psi_{2}(\boldsymbol{r}, t) d \boldsymbol{r}$ is zero by the definition of $\Psi_{2}(\boldsymbol{r}, t) d \boldsymbol{r}$ in equation (6) and orthogonality of the atomic states. The magnitude of the remaining integral $a(t)=\int \phi_{0}^{*}(\boldsymbol{r}) \Psi_{\mathrm{gs}}(\boldsymbol{r}, t) d \boldsymbol{r}$ can be estimated as follows. From equation (5) and equation (6) we obtain:
$\Psi(t)=\left(e^{-i \epsilon_{0} t}+a(t)\right) \phi_{0}+\Psi_{2}(t)$. Using the orthogonality of $\phi_{0}$ and $\Psi_{2}(t)$ and normalization of $\Psi(t)$, one obtains then: $\left|e^{-i \epsilon_{0} t}+a(t)\right|^{2}+\left\|\Psi_{2}(t)\right\|^{2}=1$, from which it follows that $2 \operatorname{Re}\left(e^{i \epsilon_{0} t} a(t)\right)+\left\|\Psi_{2}(t)\right\|^{2}+|a(t)|^{2}=0$. Since $|a(t)|=$ $O\left\|\Psi_{1}(t)\right\|$ it follows that $2 \operatorname{Re}\left(e^{i \epsilon_{0} t} a(t)\right)=O\left\|\Psi_{1}(t)\right\|^{2}$. This means that the term containing $a(t)=\int \phi_{0}^{*}(\boldsymbol{r}) \Psi_{\mathrm{gs}}(\boldsymbol{r}, t) d \boldsymbol{r}$ in the equation (8) is small compared to other terms in this equation and can be dropped. The same estimates apply in the case of the expression for the change of the velocity entropy in equation (8). We arrive, thus, at the following approximate expressions for the entropy changes:

$$
\begin{aligned}
\Delta S_{x}(t)= & 4 \operatorname{Re} e^{i \epsilon_{0} t} \int \phi_{0}^{*}(\boldsymbol{r}) \Psi_{1}(\boldsymbol{r}, t) r d \boldsymbol{r} \\
& +O\left(\left\|\Psi_{1}(\boldsymbol{r}, t)\right\|^{2},\right. \\
\Delta S_{v}(t)= & 8 \operatorname{Re} e^{i \epsilon_{0} t} \int \tilde{\phi}_{0}^{*}(\boldsymbol{q}) \tilde{\Psi}_{1}(\boldsymbol{q}, t) q^{2} d \boldsymbol{q} \\
& +O\left(\left\|\tilde{\Psi}_{1}(\boldsymbol{q}, t)\right\|^{2} .\right.
\end{aligned}
$$

The approximations we have introduced so far to simplify the exact equation equation (1) were the linearized perturbation expression (7) and its further simplification (9), obtained by replacing logarithmic factors with leading terms of their Taylor expansions, which give non-zero contributions. The figure 5 illustrates how these approximations actually work. One can see that linearized equation (7) represents exact entropies pretty accurately on the whole time interval we consider. Equation (9) represents variation of the coordinate entropy quite accurately, but is less accurate in the case of the velocity entropy. This is probably because the faster growing factor $q^{2}$ in the equation (9) for the velocity entropy makes the approximation based on the assumption that only the small vicinity near the origin contributes to the integral less accurate. In addition, as we saw above, $\tilde{\Psi}_{2}(\boldsymbol{q}, t)$ is highly oscillatory for $t \gtrsim 1.5$ o.c. We may expect the accuracy of an asymptotic estimate based on using the leading term of the expansion of the logarithmic factor in equation (7) near the origin to be less accurate for the oscillatory $\Psi_{2}$. Indeed, integrals of rapidly oscillating functions are known to be more difficult to evaluate accurately, because such integrals typically have small values, even when integrands are relatively large. Figure 5 shows, however, that even in the case of the velocity entropy we can still rely on the equation (9) at least for qualitative estimates.

To conclude this discussion we note that the equation (9) can be approximately rewritten in yet another useful form as:

$$
\begin{aligned}
& \Delta S_{x}(t)=2 \Delta\langle\Psi(\boldsymbol{r}, t)|r| \Psi(\boldsymbol{r}, t)\rangle+O\left(\left\|\Psi_{1}(\boldsymbol{r}, t)\right\|^{2},\right. \\
& \Delta S_{v}(t)=4 \Delta\left\langle\tilde{\Psi}(\boldsymbol{q}, t)\left|q^{2}\right| \tilde{\Psi}(\boldsymbol{q}, t)\right\rangle+O\left(\left\|\tilde{\Psi}_{1}(\boldsymbol{q}, t)\right\|^{2},\right.
\end{aligned}
$$

where symbol $\Delta$ on the rhs means the difference of the expectation value at time $t$ and the initial moment of time. To show that this equation is approximately valid, one can use the decomposition (5) and see, keeping the terms linear in $\Psi_{1}$, that rhs of equation (10) and lhs of equation (9) coincide. Results given by the equation (10) for the coordinate and velocity entropies are shown in the figure 7.

Being a direct consequence of the equation (9), approximation formulas (10) naturally inherit their main features. As figure 7 shows equation (7), provides a pretty accurate 

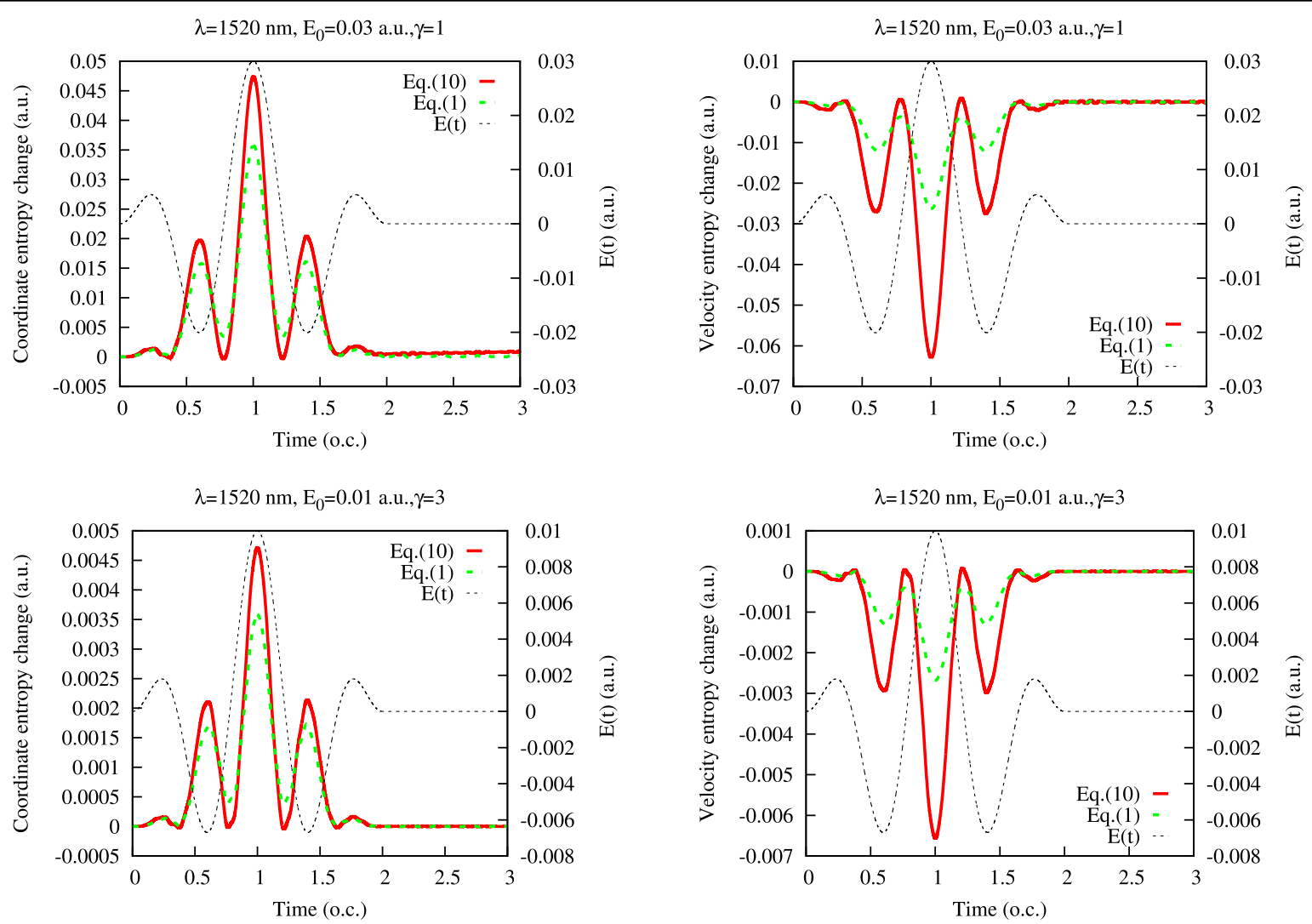

Figure 7. Exact (equation (1)) and approximate (equation (10)) entropies of the coordinate and velocity distributions.

approximation in the case of the coordinate entropy, and less accurate, but still qualitatively useful, approximation for the velocity entropy.

To see why the coordinate and velocity entropies attain, correspondingly, their local maxima and minima at the times near the local field maxima in our picture, we can take a closer look at the equation (10). We introduced above the partial wave expansion for the coordinate wave-function $\Psi(\boldsymbol{r}, t)$. Using analogous expansion for the momentum space wave-function $\tilde{\Psi}(\boldsymbol{q}, t)$ :

$$
\tilde{\Psi}(\boldsymbol{q}, t)=\sum_{l=0}^{l_{\max }} \frac{g_{l}(q, t)}{q} Y_{l 0}(\hat{\boldsymbol{q}}),
$$

we can rewrite equation (10) in the following form:

$$
\begin{aligned}
\Delta S_{x}(t)= & 2 \int_{0}^{\infty}\left(\left|f_{0}(r, t)\right|^{2}-\left|f_{0}(r, 0)\right|^{2}\right) r d r \\
& +O\left(\left\|\Psi_{1}(\boldsymbol{r}, t)\right\|^{2},\right. \\
\Delta S_{v}(t)= & 4 \int_{0}^{\infty}\left(\left|g_{0}(r, t)\right|^{2}-\left|g_{0}(r, 0)\right|^{2}\right) q^{2} d q \\
& +O\left(\left\|\tilde{\Psi}_{1}(\boldsymbol{q}, t)\right\|^{2} .\right.
\end{aligned}
$$

Evolution of the integrands in the equation (12) in time is shown in figure 8 . The processes which influence the wavefunction of the system are the excitation and ionization processes. Both these processes lead to the flow of the probability density. This flow, however manifests itself differently in the coordinate and momentum spaces. Let us consider first the case of the momentum space, and the velocity entropy for the times near the main maximum of the field $(t=1$ o.c $)$. As one can see from the figure 8, the integrand in the corresponding equation (12) has a small positive maximum at $q \approx 0.5$ a.u. and a much deeper minimum at $q \approx 1$. As the horizontal slice of the momentum distribution in figure 8 shows, it it this structure which makes the corresponding integral in equation (12), and hence the velocity entropy change at $t \approx 1$ o.c., negative. The origin of this structure can be explained qualitatively as follows. In the momentum space both ionization and excitation lead to the flow of the probability from larger to smaller $q$-values. This happens because in the momentum space both ionization and excitation processes produce components in the wave-function which are more sharply peaked at the origin than the initial state wave-function. For the excited states it follows from the trivial fact that the more a function gets extended in the coordinate space, the more narrow its Fourier transform becomes. As far as the ionization process is concerned, it produces, according to the SFA (Keldysh 1965, Popov 2004, Popruzhenko 2014b), a momentum distribution of the ionized electrons which is a Gaussian localized near the origin in the momentum space. This Gaussian again is a more narrow distribution than the momentum distribution in the ground state of hydrogen. Both these processes, therefore, lead to the effective probability flow to the region of the small $q$-values, thus making the corresponding integral in the equation (12), and the change of the velocity entropy, negative in the vicinity of $t \approx 1$ o.c.

In the coordinate space the same processes lead to a different picture. The excitation process, which as we have seen above in the figure 4 , is the dominant process for the times inside the interval of the laser pulse duration, produces 

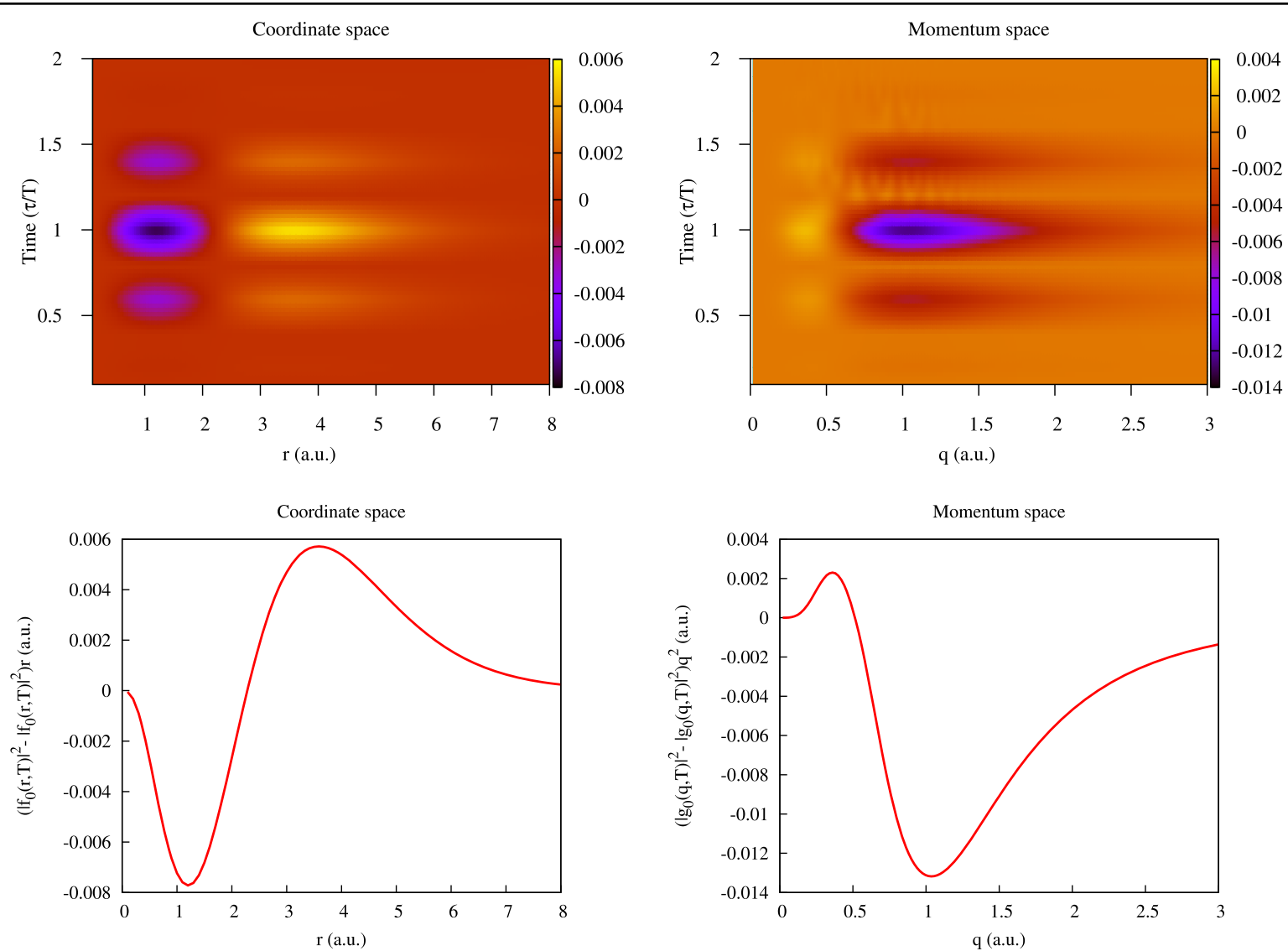

Figure 8. Integrands in equation (12) in the coordinate and momentum spaces as functions of time (top row) and for $t=1$ o.c. (bottom row) for the pulse with $E_{0}=0.03$ a.u. $\omega=0.03$ a.u .

components in the wave-function which are more extended in the coordinate space than the initial state. As far as the ionization process is concerned, the part of the wave-packet in the equation (6) describing ionized electron is localized in the coordinate space near the 'exit point'. As an estimate for the coordinate of the exit point we can use either the adiabatic tunnel exit point value $\left|z_{e}\right| \approx \frac{I_{p}}{E_{0}}$, or the FDM expression (Landsman and Keller 2015) $\left|z_{e}\right| \approx \frac{I_{p}+\sqrt{I_{p}^{2}-2 E_{0}}}{E_{0}}$, which give $\left|z_{e}\right| \approx 16$ a.u. for the electric field amplitude of 0.03 a.u. Alternatively, we can estimate the exit point as the sleeve position in figure 6 , showing coordinate distribution for the same field amplitude, which gives $\left|z_{e}\right| \approx 10$ a.u. Employing either estimate we obtain an additional component in the wave-function increasing coordinate density at large distances from the origin. The probability in the coordinate space, therefore, flows in the opposite direction, from the small to the large distances. That makes the corresponding integral in equation (12), and hence the coordinate entropy change, positive.

Other features of the behavior of the entropies in figures 1 , 2 , and 3 we have to explain, are the decrease of the coordinate entropy and increase of the velocity entropy for the times after the ionization event. As we have seen in figure 4, the excitation and ionization processes, when we consider them for the times inside the laser pulse duration, are not irreversible. After we pass the field maximum and field strength starts to decrease, the excitation and ionization probabilities diminish. In the picture we developed above that means that the probability flows in the coordinate and momentum spaces reverse their directions. The coordinate entropy, therefore, decreases while the velocity entropy increases.

These arguments can be also used to account for the visible (figures 1,2 ) net gain of the coordinate, and loss of the velocity entropies towards the end of the pulse, compared to their values in the initial ground atomic state. The excitation and ionization probabilities tend toward the end of the pulse to their physical values. As shown in figure 4, these physical values are much less than the values of the corresponding squared norms $\Psi_{\mathrm{ex}}(t), \Psi_{\text {ion }}(t)$ for the times inside the interval of the laser pulse duration. Their effect, however, is the same as we described above, excitation and ionization lead to the changes of the probability densities in the coordinate and momentum spaces which by the mechanism encapsulated in equation (12) lead to the increase of the coordinate and decrease of the velocity entropies. The magnitude of the effect at the end of the pulse is, however, much smaller, hence the smaller net increase of the coordinate entropy, and smaller decrease of the velocity entropy toward the end of the pulse, compared to the magnitude of their changes for the times inside the interval of the pulse duration. 

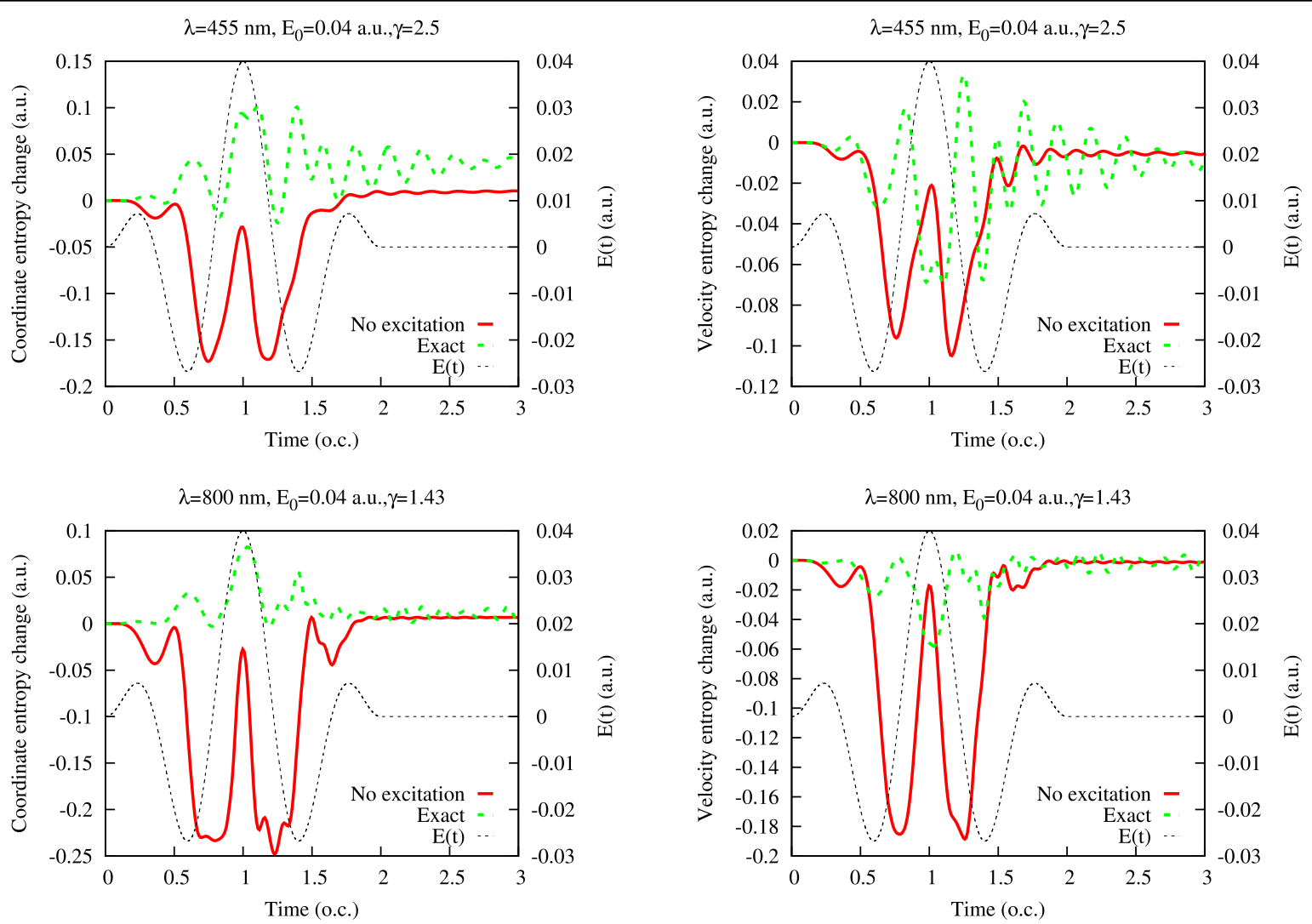

Figure 9. Coordinate and velocity entropies for the calculations using the complete TDSE wave-function and the wave-function with the excitation channel projected out.

The features of the entropies in figures 1,2 , and 3 , we must explain yet, are the oscillations clearly visible after the end of the pulse. These oscillations are due to the population trapped in the excited states after the end of the pulse. One can see from equation (7) that a component in $\Psi_{1}(\boldsymbol{r}, t)$ or $\tilde{\Psi}_{1}(\boldsymbol{q}, t)$ due to an excited state with energy $\epsilon_{1}$ leads to the terms in the entropies oscillating with time with frequency $\epsilon_{1}-\epsilon_{0}$, provided the excited state component in $\Psi_{1}$ has $s$-symmetry (otherwise the integral in equation (7) would be zero for the initial $s$-state). Amplitude of the oscillations is defined by the norm of the corresponding component of $\Psi_{1}$. To further elucidate this issue, we show in the figure 4 the coordinate and velocity entropies obtained if we project out of the wavefunction $\Psi(t)$ the component due to the excited states. In other words, to calculate entropies we use equation (1) not with the full TDSE wave-function $\Psi(t)$, but with the wave-function $\left(\hat{I}-\hat{Q}_{\mathrm{ex}}\right) \Psi(t)$, where $\hat{Q}_{\mathrm{ex}}$ is the projection operator on the excited bound states of the hydrogen atom. As figure 9 shows, using this procedure we indeed get rid of the oscillations in the entropies after the end of the pulse. Behavior of the entropies inside the pulse changes too, of course. As the figure 4 shows the excitation channel is, in fact, dominating for times inside the interval of the laser pulse duration.

Exact entropies in the figure 9 oscillate with periods of approximately $T / 4$ (the case shown in the top row of figure 9 and $T / 6$ (bottom panel of the figure)). Corresponding energy differences $\epsilon_{1}-\epsilon_{0}$ are $4 \omega \approx 0.4$ a.u. and $6 \omega \approx 0.35$ a.u. respectively ( $T$ and $\omega$ here are the optical cycle duration and the base frequency in each case). According to this estimate, the oscillations in both cases are due to the excitations of the $2 s$ state of hydrogen, which is a four photon process for $\omega=0.1$ a.u. and a six photon process for $\omega=0.057$ a.u. The latter excitation process is a much weaker one, hence the much smaller amplitude of oscillations for the case of $\omega=0.057$ a.u. in figure 9 . Oscillations of the entropies after the end of the pulse can provide, therefore, information about the excitation mechanism.

\section{Conclusion}

To conclude, we have used the information theoretic entropies of coordinate and velocity distributions to follow the evolution of the wave-function during the strong field ionization process. The property of the information theoretic entropies we employed is their ability to supply the space of probability distributions with a metric and to gauge the 'distance' between different probability distributions. This property allows to visualize conveniently the evolution of the quantum-mechanical distributions and to locate the instants of time when distributions start to evolve appreciably.

We considered both multiphoton and tunneling regimes of ionization. We saw that with increasing multiquantum parameter $K$ the entropies begin to exhibit increasingly sharper variations at times close to the local maxima of the field. Behavior of the entropies after the end of the laser pulse provides information about the excited states population and the excitation process. 
As we mentioned above, the important notion of the Keldysh tunneling time can be introduced as the time the wave-function of the system takes to adjust when the electric field is turned on instantly (McDonald et al 2013). In the present context, the non-zero tunneling time would result in the lag between the instance when wave-function of the system starts to evolve, and the maxima of the electric field. As we have seen, study of the behavior of the entropies as functions of time allows to capture the instances of the rapid evolution of the wave-function rather precisely. We can adopt a definition of the tunneling time as the lag between the local maximum of the electric field and the extremum of the coordinate or velocity entropy. As we saw above, entropy increase near the peak of the electric field is due to both excitation and ionization processes (as we noted, this division has only a qualitative character inside the laser pulse). As the top panel of the figure 4 shows, near the main maximum of the electric field the contribution of the ionization channel is of the order of about $10 \%$ of the contribution of the excitation channel. This amount would be enough to produce an appreciable lag in the entropies, if any lag in the ionization channel were present. This definition of the tunneling delay, as the lag between the contribution of the ionization channel and the electric field was adopted in (Yuan et al 2017). The contribution of the ionization channel was found using the same procedure we employed in our work, by projecting out contributions of the bound states from the solution of the TDSE. The difference with the present approach is the gauge-dependent character of this procedure. Entropies, on the other hand are gauge independent. Our calculations show no lag of any appreciable value for the entropies and hence zero tunneling delay in the framework of the definition we proposed above. We find, for instance, that for the pulse parameters in the top panel of the figure 4: $\omega=0.03$ a.u. $E_{0}=0.03$ a.u. the coordinate entropy lags behind the main maximum of the electric field by approximately 0.0016 o.c., and the velocity entropy by approximately 0.0011 o.c. These numbers are well within the numerical uncertainty of our calculations and lend support to some other results in the literature claiming essentially zero tunneling delay (Torlina et al 2015, Ni et al 2016, 2018).

Finally, we would like to emphasize the utility of the the entropy-based view. It is true, of course, that all the information about the ionization process is, in principle, available in the wave-function. It may be not easy, however, to extract and analyze this information. The wave-function (or various densities which can be derived from it) are, in general, functions of three variables and time. Evolution of such an object is not easy to follow. The entropy, on the other hand, is a single number which, as we hope we were able to demonstrate, encapsulates the key changes the system can undergo during the ionization process.

\section{Acknowledgments}

This work was supported by the Institute for Basic Science under the grant number IBS-R012-D1.

\section{ORCID iDs}

I A Ivanov (i) https://orcid.org/0000-0003-3856-1658

\section{References}

Arbo D G, Lemell C, Nagele S, Camus N, Fechner L, Krupp A, Pfeifer T, Lopez S D, Moshammer R and Burgdorfer J 2015 Phys. Rev. A 92023402

Burbea J and Rao C R 1982 J. Multivariate Anal. 12575

Corkum P B 1993 Phys. Rev. Lett. 711994

Dimitriou K I, Arbo D G, Yoshida S, Persson E and Burgdörfer J 2004 Phys. Rev. A 70 061401(R)

Dimitrovski D and Madsen L B 2015 Phys. Rev. A 91033409

Faisal F H M 1973 J. Phys. B 6 L89

Fedoryuk M V and Shabunin M I 1985 Lectures on the Theory of Functions of a Complex Variable (Moscow: Mir)

Hofmann C, Landsman A, Cirelli C, Pfeiffer A and Keller U 2013 J. Phys. B 46125601

Hu B, Liu J and Chen S G 1997 Phys. Lett. A 236533

Ivanov I A, Nam C H and Kim K T 2017 Sci. Rep. 739919

Ivanov I A 2014 Phys. Rev. A 90013418

Ivanov I A and Kheifets A S 2013 Phys. Rev. A 87033407

Keil T, Popruzhenko S V and Bauer D 2016 Phys. Rev. Lett. 117 243003

Keldysh L V 1965 Sov. Phys. - JETP 201307

Krausz F and Ivanov M 2009 Rev. Mod. Phys. 81163

Kulander K C, Schafer K J and Krause J L 1993 Dynamics of ShortPulse Excitation, Ionization and Harmonic Conversion (Berlin: Springer)

Landau L D and Lifshitz E M 1977 Quantum Mechanics (Oxford: Pergamon)

Landau L D and Lifshitz E M 1980 Statistical Physics (Oxford: Pergamon)

Landsman A S and Keller U 2015 Phys. Rep. 5471

Leipnik R 1959 Inf. Control 264

Lewenstein M, Balcou P, Ivanov M Y, L'Huillier A and Corkum P B 1994 Phys. Rev. A 492117

Majorosi S, Benedict M G and Czirják A 2017 Phys. Rev. A 96 043412

McDonald C R, Orlando G, Vampa G and Brabec T 2013 Phys. Rev. Lett. 111090405

Ni H, Saalmann U and Rost J M 2016 Phys. Rev. Lett. 117 023002

Ni H, Saalmann U and Rost J M 2018 Phys. Rev. A 97013426

Perelomov A M, Popov V S and Terentiev M V 1966 Sov. Phys. JETP 23924

Pfeiffer A N, Cirelli C, Landsman A S, Smolarski M, Dimitrovski D, Madsen L B and Keller U 2012 Phys. Rev. Lett. 109 083002

Pisanty E and Ivanov M 2016 Phys. Rev. A 93043408

Popov V S 2004 Phys.-Usp. 47855

Popruzhenko S V 2014a Sov. Phys. - JETP 118 580-6

Popruzhenko S V 2014b J. Phys. B: At. Mol. Opt. Phys. 47 204001

Reiss H R 1980 Phys. Rev. A 221786

Shannon C 1948 Bell Syst. Tech. J. 27379

Shvetsov-Shilovski N I, Dimitrovski D and Madsen L B 2012 Phys. Rev. A 85023428

Sobelman I I 1972 Introduction to the Theory of Atomic Spectra (Oxford: Pergamon)

Torlina L et al 2015 Nat. Phys. 11503

Yuan M, Xin P, Chu T and Liu H 2017 Opt. Express 2523493

Zimmermann T, Mishra S, Doran B R, Gordon D F and Landsman A S 2016 Phys. Rev. Lett. 116233603 